\title{
Die Akutbehandlung der Schizophrenie
}

\author{
Rüdiger Holzbach \\ Universitätsklinikum Hamburg-Eppendorf, Psychosoziales Zentrum, Klinik für Psychiatrie und \\ Psychotherapie
}

psychoneuro 2003; 29 (11): 522-526

Die angemessene Behandlung einer akuten Schizophrenie ist für den Betroffenen unter Umständen eine lebensentscheidende Weichenstellung. Es besteht die Gefahr, dass ein an einer Schizophrenie Erkrankter aus dem Erwerbsleben ausscheidet und sozial absteigt. Deshalb sollten neben der Pharmakotherapie auch weitere Behandlungselemente zum Tragen kommen: Psychoedukation, Auslöseridentifikation und Beeinflussung verlaufsmodifizierender Faktoren. Je nach Krankheitsphase und Krankheitsschwere sind unterschiedliche Gewichtungen der Behandlungselemente notwendig. Die Behandlung muss frühestmöglich beginnen und mit den wirksamsten Mitteln geschehen, da das anfänglich Versäumte später nur schwer nachzuholen ist. Bei der Pharmakotherapie sollten, wenn bisher eingesetzte klassische Neuroleptika nicht sehr gut vertragen wurden, immer neuere atypische Neuroleptika eingesetzt werden. Bei Ersterkrankten empfiehlt sich die Behandlung mit atypischen Neuroleptika von Anfang an.

$\mathrm{M}$ it der Diagnose Schizophrenie wird eine Gruppe von Krankheitsbildern zusammengefasst, die ähnliche Grundsymptome und ein Spektrum an akzessorischen Symptomen aufweisen. Bis heute ist die genaue Ursache dieser Erkrankungen ungeklärt. Allgemein wird von einer hirnorganischen Verursachung ausgegangen. Eine vererbbare Vulnerabilität für diese Erkrankung kann zusammen mit lebensgeschichtlichen Belastungen und diskreten zerebralen Schädigungen (z.B. prä- und/oder postnatale Virusinfektionen) zum Ausbruch der Erkrankung führen. Der häufigste Schizophrenie-Typ ist die paranoide Schizophrenie, bei der das Wahnerleben das führende Symptom darstellt. In den akuten Phasen stehen meistens die produktiv psychotischen Symptome (Wahr- nehmungsstörungen, formale und inhaltliche Denkstörungen und gestörtes Ich-Erleben) im Vordergrund, bei chronischen Verläufen finden sich eine Negativ- bzw. Minussymptomatik, die auch als Residualsyndrom bezeichnet wird, gekennzeichnet durch abgeflachten Affekt, verminderten Antrieb, Denkverarmung und weitere Leistungseinbußen im Bereich Kognition, Gedächtnis und körperlicher Leistungsfähigkeit.

\section{Diagnosestellung}

Schizophrene Erkrankungen können alle Bereiche der Wahrnehmung, des Denkens, des Fühlens und des Ich-Erlebens sowie den Antrieb betreffen. In Tabelle 1 sind die allgemeinen Diagnosekriterien gemäß ICD-10 zusammengefasst. Über die Schwierigkeit der Früherkennung berichtet

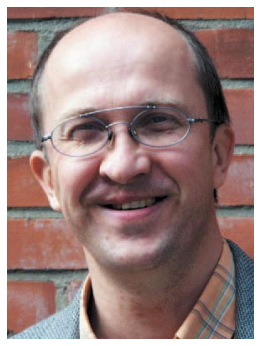

Rüdiger Holzbach
Hambrecht in diesem Heft. Die Differenzialdiagnostik kann unter Umständen sehr schwierig sein, da nahezu alle psychopathologischen Auffälligkeiten im Rahmen einer Schizophrenie auftreten können, andererseits besteht die Gefahr, dass aufgrund einzelner Symptome (z.B. Halluzinationen) voreilig auf eine Schizophrenie geschlossen wird. In Rahmen dieses Beitrages kann nur empfohlen werden, sich im Zweifelsfall konsiliarisch Rat zu holen. Obwohl bei schizophrenen Erkrankungen eine frühzeitige Behandlung zu empfehlen ist $(4,7)$, sollte nicht „auf Verdacht“ behandelt werden.

\section{Grundüberlegungen \\ zur Behandlung}

In den letzten Jahren sind die volkswirtschaftlichen Kosten von Erkrankungen zunehmend Gegenstand der Betrachtung geworden. Die Schizophrenie, obwohl selten (Lebenszeitprävalenz ca. 1\%), ist eine sehr teure Erkrankung. Dies wäre ein zu vernachlässigender Aspekt, wenn durch zwar teure, aber effektive Behandlungen den Betroffenen eine Gesundung bzw. trotz Erkrankung eine hohe Lebensqualität ermöglicht würde. Die Kosten entstehen aber leider dadurch, dass ein Großteil der an Schizophrenie Erkrankten aus dem Erwerbsleben 
ausscheidet und sozial absteigt. In der Regel vergehen sechs wichtige Jahre, bis die Erkrankung behandelt wird (6). Da der Erkrankungsgipfel im jungen Erwachsenenalter liegt (Männer erkranken früher als Frauen), werden bereits durch die Prodromalsymptome (sozialer Rückzug, uncharakteristische Verhaltensänderungen, Leistungsminderung, fremdartige Ideen, unspezifische affektive Änderungen) Biografien negativ beeinflusst, und der Aufbau einer eigenen Existenz gelingt nur eingeschränkt oder nicht. Um dies zu verhindern, muss die Behandlung frühestmöglich beginnen und mit den wirksamsten Mitteln geschehen, da das anfänglich Versäumte später nur schwer nachzuholen ist.

\section{Grundprinzipien \\ der Behandlung}

Die Behandlung von an Schizophrenie erkrankten Menschen sollte immer die folgenden vier Bereiche berücksichtigen: Informationsvermittlung (Psychoedukation), Auslöseridentifikation, Beeinflussung verlaufsmodifizierender Faktoren und medikamentöse Behandlung. Je nach Krankheitsphase und Krankheitsschwere sind unterschiedliche Gewichtungen der Behandlungselemente notwendig.

\section{Informationsvermittlung (Psychoedukation)}

Ähnlich wie zum Beispiel bei der Schulung von Diabetikern schon lange etabliert, wird zunehmend auch im Bereich psychischer Störungen versucht, Betroffene zu Experten in Sachen der eigenen Erkrankung auszubilden. Diese so genannten Psychoedukationsprogramme haben sich auch im Bereich der Schizophrenie bewährt (11). Neben dem gängigen Krankheitskonzept, typischen Symptomen und den möglichen Verlaufsformen wird vor allem über die Behandlungsmöglichkeiten, Aufklärung über Nebenwirkungen und Umgang mit Neuroleptika, Frühwarnzeichen für einen neuen Krankheitsschub, Umgang mit Krankheitssymptomen und eigene Einflussmöglichkeiten auf den Krankheitsverlauf eingegangen. Wo immer möglich, sollte dies in Gruppen geschehen. Dabei können sich die Gruppenteilnehmer gegenseitig austauschen und ihre Erfahrungen aufgegriffen werden. Reiner „Frontalunterricht“ ist zu vermeiden.

\section{Auslöseridentifikation}

Menschen, die erkranken, suchen nach einer Erklärung für ihre Erkrankung. Zumeist wird in kurzfristigen zeitlichen Rahmen gedacht, bei psychischen Erkrankungen wird aber auch aufgrund allgemein verbreiteter (tiefen-)psychologischer Modelle die Kindheit und Jugendzeit miteinbezogen. Dieser Wunsch nach Erklärung sollte genutzt werden, insbesondere da auslösende Faktoren das Risiko bergen, zu aufrechterhaltenden Faktoren zu werden.

Wie bereits erwähnt, ist die gängige Hypothese zur Schizophrenieentstehung die einer multifaktoriellen Genese. Dazu gehören nicht beeinflussbare Faktoren, wie genetisches Risiko bei familiär gehäuft auftretenden Erkrankungen aus dem schizophrenen Formenkreis (bei erstgradig Verwandten 10-15fach höheres Risiko [5]), nur bedingt be- einflussbare, wie erworbene (minimale) Hirnfunktionsstörungen, und veränderbare Faktoren, wie Stress und exogene Noxen. Obwohl nicht immer auslösende vermeidbare Belastungen gefunden werden können, sollte einiges Augenmerk auf diese Punkte gerichtet werden, da diese auch bei den aufrechterhaltenden Faktoren eine Rolle spielen (s. u.).

Sehr häufig wird bei jungen Ersterkrankten im Vorfeld ein CannabisKonsum festgestellt. Allerdings ist noch unklar, inwieweit dies Zufall ist, ob es sich um eine Selbstbehandlung in der Prodromalphase (s. „Grundüberlegungen zur Behandlung“) handelt oder ob Cannabis einen auslösenden Faktor darstellt. Da Patienten, die im Verlauf weiter Cannabis konsumieren, einen ungünstigeren Verlauf nehmen $(1,3)$, sollte von einem weiteren Cannabis-Konsum strikt abgeraten werden. Dies gilt auch für alle anderen Suchtmittel. Lediglich Nikotin und Benzodiazepine dürften bei der Auslösung schizophrener Erkrankungen keine Bedeutung haben, beeinflussen aber den Verlauf (s. u.).

\section{Tab. 1 Diagnostische Leitlinien der Schizophrenie}

\section{Mindestens ein eindeutiges Symptom (oder mindestens zwei weniger eindeutige) der Gruppe A:}

$$
\begin{aligned}
& \text { Gruppe A Gedankenlautwerden, Gedankeneingebung, Gedankenentzug, Gedankenaus- } \\
& \text { breitung } \\
& \text { - Kontrollwahn, Beeinflussungswahn, Gefühl des Gemachten, deutlich bezogen } \\
& \text { auf Körper- oder Gliederbewegungen oder bestimmte Gedanken, Tätigkeiten } \\
& \text { oder Empfindungen; Wahnwahrnehmungen } \\
& \text { - Kommentierende oder dialogische Stimmen, die über den Betroffenen und } \\
& \text { sein Verhalten sprechen oder ihm befehlen } \\
& \text { - Anhaltender, kulturell unangemessener oder völlig unrealistischer Wahn }
\end{aligned}
$$

\section{Oder mindestens zwei Symptome der Gruppe B:}

Gruppe B - Anhaltende Halluzination eines oder mehrerer Sinne, täglich auftretend, begleitet von flüchtigen oder undeutlich ausgebildeten Wahngedanken ohne deutliche affektive Beteiligung, oder begleitet von anhaltenden überwertigen Ideen

- Gedankenabreißen oder Einschiebungen, mit der Folge Zerfahrenheit oder Danebenreden

- Katatone Symptome wie Erregung, Haltungsstereotypien, Flexibilitas cerea, Negativismus, Mutismus, Stupor

- Minussymptome wie auffällige Apathie, Sprachverarmung, Affektverarmung, Verflachung oder inadäquate Affekte verbunden mit sozialem Rückzug und verminderter sozialer Leistungsfähigkeit

Weiterhin muss erfüllt sein: Die Symptome bestehen mindestens vier Wochen (die meiste Zeit) und die Symptome sind durch keine organische Störung zu erklären.

(modifiziert nach 2) 
Sowohl bei dem Ausbruch der Erkrankungen als auch bei Rezidiven finden sich im Vorfeld häufig belastende Veränderungen (z.B. Wohnortwechsel, Ausbildungsbeginn, Beziehungsverlust). Vermutlich reichen in diesen Phasen die Ressourcen und Kompensationsmechanismen nicht mehr aus, um die „Reizüberflutung" zu begrenzen und zwischen real und irreal zu unterscheiden.

In diesen Kontext gehört auch emotionaler Stress, der bei manchen Familien aus dem dort üblichen Interaktionstil mit „high-expressedemotions“ resultiert. In diesen Familien werden Emotionen und Anschauungen sehr intensiv ausge- tauscht und können so ebenfalls die Patienten überfordern.

Verlaufsmodifizierende Faktoren

Bei den verlaufsmodifizierenden Faktoren spielen einerseits aufrechterhaltende Faktoren eine Rolle (negativer Einfluss), andererseits interessieren hier positiv beeinflussende Maßnahmen wie medikamentöse Behandlung (s. u.) und Behandlung der Negativsymptomatik bzw. des Residualsyndromes.

Minimierung negativer Einflüsse Alle bereits unter den auslösenden Faktoren genannten Punkte haben einen negativen Einfluss auf den Verlauf einer schizophrenen Erkran- kung. Bei den illegalen Drogen und bei Alkohol addiert sich zum Risiko der Triggerung eines neuerlichen Krankheitsschubes auch die reduzierte Zuverlässigkeit bei der Medikamenteneinnahme, was wiederum nochmals die Rezidivgefahr erhöht. Nikotin kann zu einer verstärkten Metabolisierung der Neuroleptika führen und durch daraus resultierende niedrigere Plasmaspiegel eine geringere antipsychotische Wirksamkeit hervorrufen. Benzodiazepine verstärken die dämpfende Wirkung einiger Neuroleptika. Dies kann bei akut erregten Patienten sinnvoll sein. In der Langzeitanwendung dieser Kombinationsbehandlung treten im Verlauf Symptome

\section{Tab. 2 Übersicht einiger wichtiger Neuroleptika}

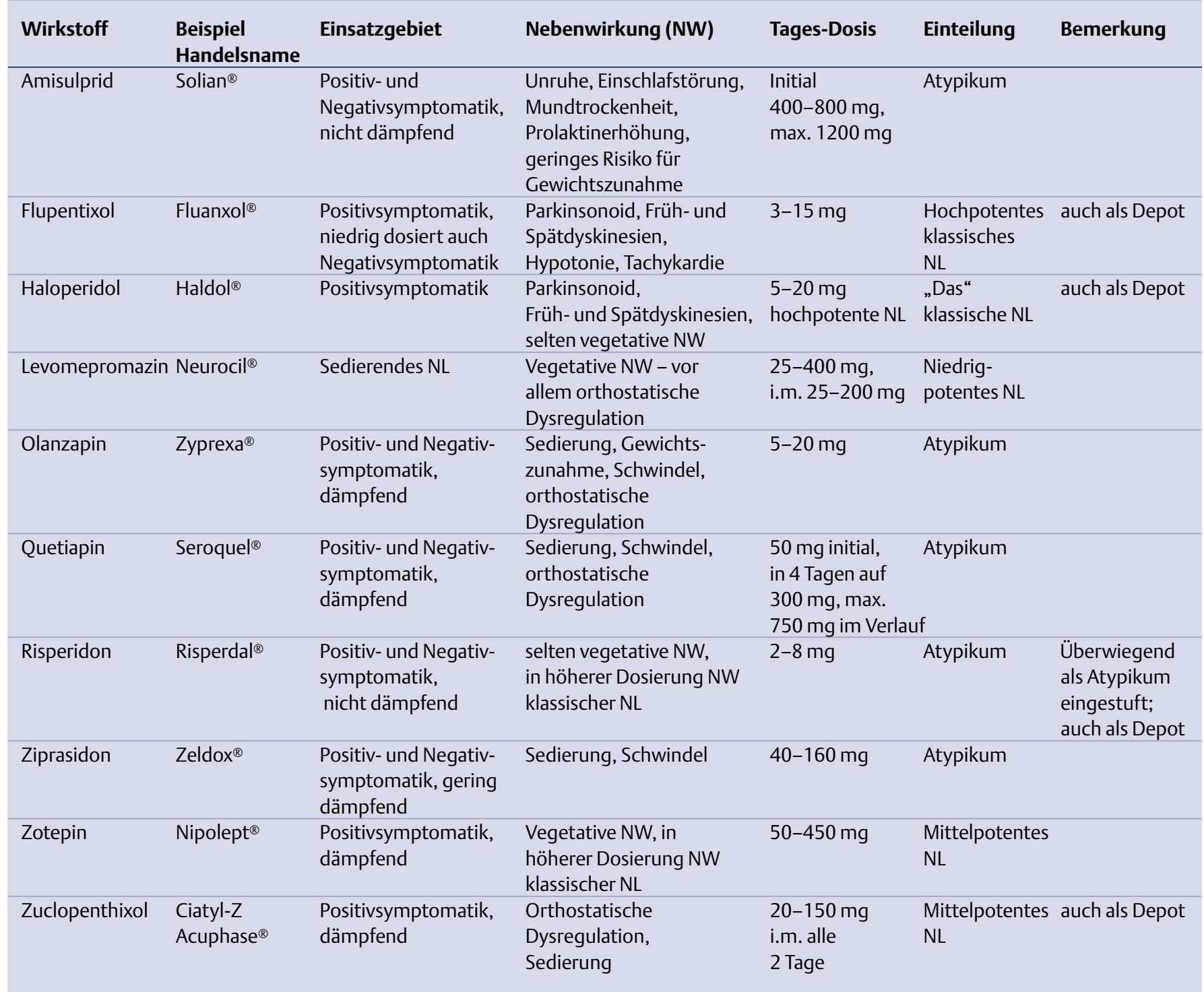


der Abhängigkeit von Benzodiazepinen auf (8), wodurch das Residualsyndrom verstärkt wird.

Die intensiv ausgedrückten Emotionen und Meinungen in ,high-expressed-emotions“-Familien oder Umfeld führen zu häufigeren Rezidiven. Berichten Patienten über ein entsprechendes Umfeld oder wird dies bei dem obligaten Beratungsgespräch des Umfeldes deutlich, so sollten in weiteren Familiengesprächen diese Interaktionsmuster verändert werden oder ggf. der Patient unterstützt werden, sich gegen diese Umfeld besser abzugrenzen oder daraus zu lösen.

Auch schwerwiegende Belastungen können zu einer neuerlichen Krankheitsmanifestation mit produktiv-psychotischen Symptomen führen. Der darüber informierte Patient sollte bei absehbaren Belastungen sich rechtzeitig Unterstützung suchen, um die Belastungen abzumildern. Auch eine erhöhte neuroleptische Medikation sollte erwogen werden.

Fehlende Compliance wirkt sich bei schizophrenen Erkrankungen mit einer Rezidivwahrscheinlichkeit von rund $66 \%$ fatal aus. Neben der zuverlässigen Medikamenteneinnahme gehört auch die Berücksichtigung der hier genannten Punkte zur Compliance dazu.

\section{Stärkung positiver Einflüsse}

Hier ist zunächst die Pharmakotherapie zu nennen (s.u.). Patienten mit einem Residualsyndrom und einer Unterstimulation durch das Umfeld zeigen einen ungünstigen Verlauf. Neben der Pharmakotherapie gehören deshalb flankierende psychosoziale Maßnahmen zum Behandlungsstandard. Hier besteht mittlerweile ein reichhaltiges Spektrum, sowohl in Hinblick auf die Unterbringung (von Betreuung im eigenen Wohnraum bis zu soziotherapeutischen Wohngruppen), die Beschäftigung (von Ergo- und Arbeitstherapie bis zur beruflichen Reha) als auch auf die therapeutischen Verfahren (sozio-, verhaltens-, familien- und pharmakotherapeutisch) und Settings (ambulant, teilstationär, stationär).

Schizophrene Patienten können sowohl durch Positiv- wie Negativ- symptome auf Unverständnis oder Konflikte mit ihrem Umfeld stoßen. Hier können vermittelnde Gespräche zusammen mit ärztlichen oder soziotherapeutischen Behandlern und Arbeitskollegen, Nachbarn oder Familie und Freunden Missverständnisse oder Fehleinschätzungen korrigieren und die weitere Hilfe des Umfeldes sichern. Die Bedeutung eines stabilen und verlässlichen (unterstützenden) Umfeldes kann gar nicht hoch genug eingeschätzt werden - deshalb sollte hier kein Aufwand gescheut werden.

\section{Pharmakotherapie}

Neben den erwähnten psychosozialen Maßnahmen ist die Pharmakotherapie das zweite unverzichtbare Standbein der Schizophreniebehandlung. Oft werden durch eine Pharmakotherapie die weiterführenden psychosozialen Maßnahmen erst möglich.

Die dazu eingesetzten Neuroleptika (NL) lassen sich einerseits in niedrig-, mittel- und hochpotente Neuroleptika einteilen, andererseits nach ihrem Nebenwirkungsspektrum und der Wirksamkeit auf Negativsymptome in typische (klassische) und atypische (neuere) Neuroleptika (Tab. 2). Die besonders gefürchteten Nebenwirkungen klassischer NL sind Parkinsonoid, Früh- und Spätdyskinesien.

NL in der Akutbehandlung der Schizophrenie sollen in erster Linie gut gegen die produktiv-psychotischen Symptome wirken und gut verträglich sein. In zweiter Linie ist die Möglichkeit einer Fortführung der Therapie zur Rezidivprophylaxe ohne kognitive Beeinträchtigung und ggf. Wirksamkeit gegen ein Residualsyndrom anzustreben. Darüber hinaus stellt sich in der Akutbehandlung die Frage, ob eine zusätzliche Dämpfung bei erregten Patienten erforderlich ist.

Um die Vorgehensweise bei der Akutbehandlung konkret darzustellen, sind im Nachfolgenden zwei verschiedene Symptomlagen zugrunde gelegt.

\section{Beginnend akut psychotische Patienten}

In diesem Beispiel bemerkt der Patient selbst bei sich vermehrt psy- chotische Symptome, entweder unter einer konstant genommenen rezidivprophylaktischen neuroleptischen Therapie bei vermehrter psychosozialer Belastung oder nachdem er seine Medikation im Vorfeld abgesetzt hatte. Entsprechend sollten die bisherigen NL erhöht oder wieder angesetzt werden, wenn sie bisher gut vertragen wurden. Insbesondere bei Patienten, die im Vorfeld die NL abgesetzt haben, sollte viel Mühe darauf verwendet werden, zu verstehen, weshalb sie die Medikation abgesetzt haben. Häufig spielt nicht nur die Hoffnung hinein, die Medikation nicht mehr zu brauchen, sondern auch eine Unzufriedenheit mit der Medikation. Dementsprechend sollte rechtzeitig an eine Medikamentenumstellung gedacht werden. In der Regel sollte heute auf die zwar teureren, aber besser verträglichen atypischen NL eingestellt werden. Das subjektive Wohlbefinden der Patienten darunter ist höher (10) und die geringere Rezidivrate sowie die bessere Reintegrationsfähigkeit senken die Gesamtkosten der Behandlung sogar (9). Die Wahl des Atypikums richtet sich wiederum nach dem jeweiligen Nebenwirkungsspektrum - z.B. wird die Gefahr der Gewichtszunahme oder der gestörten Libido mittelfristig von vielen Patienten nicht toleriert.

\section{Erregte akut psychotische Patienten}

Nach einem mehr oder weniger langen psychotischen „anschoppen“ manifestiert sich das psychotische Erleben meist recht akut. Die Patienten fühlen sich beobachtet und verfolgt, haben Angst und beziehen immer mehr Ereignisse in ihrem Umfeld mit in das Wahnerleben ein. Sie sind innerlich und motorisch unruhig, ängstlich, im Kontakt nur schwer zu erreichen und unter Umständen aggressiv-angespannt. $\mathrm{Zu}$ meist läuft diese Konstellation auf eine stationäre Behandlung hinaus, eine Dämpfung des Patienten ist in seinem und im Interesse des Umfeldes anzustreben. Prinzipiell stehen drei Vorgehensweisen zur Wahl:

- Kombination eines atypischen NL mit einem Benzodiazepin - Kombination eines (klassischen) 
hochpotenten NL mit einem niedrigpotenten NL oder einem Benzodiazepin

- Hochdosierte Gabe eines mittelpotenten NL.

Alle Behandlungsoptionen bieten Vor- und Nachteile. Je seltener man Akutbehandlungen schizophrener Patienten durchführt, umso mehr sollte man sich auf nur eine Methode konzentrieren und sich damit auskennen. Ansonsten kann nach den folgenden Kriterien abgewogen werden:

Auf die Kombination eines atypischen NL mit einem Benzodiazepin sollte immer dann zurückgegriffen werden, wenn Ersterkrankte nicht durch unangenehme Nebenwirkungen typischer NL einer weiteren Pharmakotherapie gegenüber abgeschreckt werden sollen. Dazu würde auch gehören, zur Dämpfung ein Benzodiazepin zu verwenden, da auch niederpotente NL klassische Nebenwirkungen hervorrufen können. Es ist immer noch umstritten, ob atypische NL langsamer ihre antipsychotische Wirkung entfalten als klassische NL. Vermutlich ist dies im klinischen Alltag ein Dosierungsproblem, da entsprechende Studien diesen Unterschied nicht zeigen. Eine entsprechende konkrete Behandlung wäre z.B. 15-20 mg Olanzapin/Tag und 10-20 mg Diazepam/Tag.

Die Kombination eines klassischen hochpotenten NL mit einem niedrigpotenten NL oder einem Benzodiazepin bietet sich vor allem an, wenn bei dem Patienten aus Vorbehandlungen eine gute Verträglichkeit der hochpotenten NL bekannt ist und ein schneller Wirkeintritt erzielt werden soll. Zur Entscheidung zwischen niedrigpotenten NL und einem Benzodiazepin zur Dämpfung liegen keine ausreichenden wissenschaftlichen Untersuchungen vor. Für die Benzodiazepine sprechen die geringere Nebenwirkung auf den Blutdruck, dagegen eine eventuell bestehende Suchtvorgeschichte. Die Behandlung eines akut erregten Patienten würde dann z.B. mit 10-20 mg Haloperidol/Tag und Levomepromazin 50-150 mg/Tag oder 10-20 mg Diazepam/Tag erfolgen.
Der Vorteil einer hochdosierten Behandlung mit einem mittelpotenten Neuroleptikum in der Akutbehandlung liegt in der besonderen Darreichungsform von Zuclopenthixolacetat als kurzwirksames Depot mit zwei bis drei Tagen Wirkungsdauer für Patienten, die eine orale Medikation ablehnen. Die konkrete Anwendungsempfehlung lautet 5-150 mg Zuclopenthixolacetat i.m.

\section{- Fazit}

In der Akuttherapie der Schizophrenie ist zu beachten, dass die Therapie nicht nur frühzeitig und rasch wirksam durchzuführen ist, sondern auch für den Patienten als angemessen empfunden wird. Eine rein medikamentöse Therapie ist hierbei nicht ausreichend. Besonders bei ersterkrankten, aber auch bei krankheitserfahrenen Patienten, die eine erneute Exazerbation erleiden, müssen psychoedukative Maßnahmen angewandt, Auslöser identifiziert und Verhalten modifiziert werden, damit die Erkrankung zuverlässig behandelt werden kann und eine notwendige Langzeittherapie bessere Erfolgschancen hat. Psychotische Patienten, die sich beim Hausarzt vorstellen, sollten deshalb in jedem Fall auch von einem Nervenarzt gesehen werden.

For a patient developing acute schizophrenia, the provision of appropriate treatment may be of decisive importance in setting the points for the future. There is a real danger that a person suffering from schizophrenia may be deprived of gainful employment and undergo social decline. For this reason, drug treatment must be flanked by further therapeutic options such as: psychoeducation, identification of triggering mechanisms, and acting on factors modifying the course of the illness. Depending on the phase and severity of the illness, the various aspects of treatment will require different emphasis. Treatment must be initiated as early as possible, and the most effective agents used, since it is extremely difficult to compensate for initial failings. With regard to pharmacotherapy, if previously used classical neuroleptic agents have not been well tolerated, more modern atypical neuroleptics should always be employed. In patients developing schizophrenia for the first time, treatment with atypical neuroleptics is to be recommended right from the beginning.

\section{Key Words}

Schizophrenia - acute treatment

\section{Literatur}

1. Caspari D. Cannabis and schizophrenia: results of a follow-up study. Eur Arch Psychiatry Clin Neurosci 1999; 249: 45-49

2. Dilling H, Mombour W, Schmidt MH. Internationale Klassifikation psychischer Störungen. ICD-10 Kapitel V (F). Verlag Hans Huber, 2. Auflage 1993

3. Drake RE, Brunette MF. Complications of severe mental illness related to alcohol and drug use disorders. In: Galanter M (Hrsg.): Recent developments in alcoholism: the consequences of alcohol. Vol. 14. New York, Plenum Press, 1998; 285-299

4. Drake RJ, Haley CJ, Akhtar S, Lewis SW. Causes and consequences of duration of untreated psychosis in schizophrenia. Br J Psychiatry 2000 ; $177: 511-515$

5. Gottesmann El, Bertelsen A. Confirming an expressed genotype for schizophrenia. Arch Gen Psychiatry 1989; 46: 867-872

6. Häfner $\mathrm{H}$, an der Heiden $\mathrm{W}$, Hambrecht M, Riecher-Rössler A, Maurer K, Löffler W, Fätkenheuer B. Ein Kapitel systematischer Schizophrenieforschung - Die Suche nach kausalen Erklärungen für den Geschlechtsunterschied im Ersterkrankungsalter. Nervenarzt 1993; 64: 706-716

7. Harrigan SM, McGorry PD, Krstev H. Does treatment delay in first-episode psychosis really matter? Psychol Med 2003; 33 97-110

8. Holzbach R. Benzodiazepinabhängigkeit. Abhängigkeiten 2000; 2: 5-16

9. Lambert M. Pharmakoökonomie. In: Naber D, Lambert M, Krausz M, Haasen C (Hrsg.). Atypische Neuroleptika in der Behandlung schizophrener Patienten. Bremen, Uni-med, 2000; 268-273

10. Naber D, Moritz S, Lambert M, Pajonk F, Holzbach R, Maß R, Andresen B. Schizophrenic psychopathology and subjective wellbeing under neuroleptics. Schizophrenia Research 2001; 50: 82-90

11. Pekkala E, Merinder L. Psychoeducation for schizophrenia. Cochrane Database Syst Rev. 2002; 2: CD002831

\section{Korrespondenzadrese:}

Dr. R. Holzbach

Universitätsklinikum Hamburg-Eppendorf Psychosoziales Zentrum

Klinik für Psychiatrie und Psychotherapie

Martinistr. 52

20246 Hamburg

e-mail: holzbach@uke.uni-hamburg.de 